\title{
Predictors of pathologic complete response to neoadjuvant treatment in HER2-overexpressing breast cancer: a retrospective analysis using real-world data
}

\author{
Isabel Saffie Vega ${ }^{1,2}$, Jorge Sapunar Zenteno ${ }^{2,3}$, Felipe Buscaglia Fernandez ${ }^{4}$, Felipe Reyes Cosmelli ${ }^{5}$, Rodrigo Lagos Chavez ${ }^{2}$ and \\ Badir Chahuán Manzur ${ }^{1}$
}

\begin{abstract}
${ }^{1}$ Breast Oncologic and Reconstructive Surgery Unit, Instituto Oncológico Fundación Arturo López Pérez, Santiago 7500691, Chile 2Epidemiologic and Clinical Research Unit, Department of Cancer Research, Instituto Oncológico Fundación Arturo López Pérez, Santiago 7500691, Chile ${ }^{3}$ Department of Internal Medicine, School of Medicine, Universidad de La Frontera, Temuco 4811230, Chile ${ }^{4}$ Pathology Anatomy Unit, Instituto Oncológico Fundación Arturo López Pérez, Santiago 7500691, Chile

${ }^{5}$ Medical Oncology Unit, Instituto Oncológico Fundación Arturo López Pérez, Santiago 7500691, Chile
\end{abstract}

\section{Abstract}

The human epidermal growth factor receptor 2 (ERBB2, HER2 or HER2/neu) is a transmembrane tyrosine kinase receptor that is overexpressed in approximately $20 \%$ of breast cancers. The use of the anti-HER2 monoclonal antibodies Pertuzumab and Trastuzumab in association with chemotherapy has achieved a higher percentage of pathologic complete response ( $p C R$ ) than conventional chemotherapy. The purpose of our study was to identify factors that could affect the therapeutic response of patients with breast cancer and HER2 overexpression treated with cytotoxic chemotherapy plus double HER2 blockade in neoadjuvant setting at Fundación Arturo López Pérez (FALP). A case-control study was designed to evaluate the effect of clinical and histopathological variables on the response to neoadjuvant therapy. Ninety-four women with non-metastatic breast cancer and HER2 overexpression received neoadjuvant combination chemotherapy with Trastuzumab and Pertuzumab at FALP during the period 2017-2020. Seventy percent of patients achieved $\mathrm{pCR}$, and in the group of hormone receptor negative patients, $89 \%$ of patients achieved $\mathrm{pCR}$. Different variables were analysed trying to look for clinicopathological predictors of complete response. This study provides us with real-world data on the efficacy of using this treatment combination in our population of HER2-overexpressing breast cancer patients.

Keywords: breast cancer, HER2 positive, neoadjuvant chemotherapy, pathologic complete response, trastuzumab, pertuzumab

\section{Introduction}

Breast cancer is the most commonly diagnosed malignancy, regardless of the level of development of regions and countries [1]. One in eight women will have breast cancer in her lifetime and it is the leading cause of cancer mortality in the Chilean population with

Correspondence to: Isabel Saffie Vega Email: isabel.saffie@falp.org

ecancer 2022, 16:1338

https://doi.org/10.3332/ecancer.2022.1338

Published: 06/01/2022

Received: 09/08/2021

Publication costs for this article were supported by ecancer (UK Charity number 1176307).

Copyright: (c) the authors; licensee ecancermedicalscience. This is an Open Access article distributed under the terms of the Creative Commons Attribution License (http:// creativecommons.org/licenses/by/4.0), which permits unrestricted use, distribution, and reproduction in any medium, provided the original work is properly cited. 
an age-standardised rate of 10.2 per 100,000 women [2]. The World Health Organization considers it one of the main public health problems in developed and developing countries.

Today, breast cancer is classified into different molecular subtypes that correlate closely with the presence or absence of immunohistochemical markers: hormone receptor positive/HER2 negative, HER2 positive and triple negative in which case there is no expression of hormone receptors or HER2 protein. Each corresponds to approximately $70 \%, 20 \%$ and $10 \%$ of breast cancers, respectively. These breast cancer subtypes have different risk profiles and treatment strategies [3].

Human epidermal growth factor receptor 2 (ERBB2, HER2 or HER2/neu) is a molecular target consisting of a transmembrane tyrosine kinaselike receptor. This epidermal growth factor is overexpressed or amplified in approximately $20 \%$ of breast cancers and has been associated with worse prognosis in the absence of targeted systemic therapy [4]. HER2-overexpressing tumours benefit from anti-HER2 target therapies such as the monoclonal antibodies Trastuzumab and Pertuzumab, as well as small molecule tyrosine kinase inhibitors such as Lapatinib and Neratinib among others. These treatments have prolonged progression-free survival and overall survival in the subgroup of patients with metastatic breast cancer and also improved pathologic complete response ( $\mathrm{pCR}$ ) rates in patients with less advanced stages receiving neoadjuvant therapy [5].

The clinical benefit of combining Trastuzumab and Pertuzumab with chemotherapy was initially demonstrated in the CLEOPATRA study where there was a significant increase in progression-free survival and overall survival in patients with HER2-overexpressing tumours and metastatic disease [8, 9].

Based on these results, the use of Pertuzumab, Trastuzumab and chemotherapy was evaluated as neoadjuvant therapy in patients with HER2 disease in two phase 2 studies [6, 7]. In these studies, a higher percentage of patients achieved pCR with combined anti-HER2 treatment added to conventional chemotherapy.

The results of combined anti-HER2 treatment in HER2-overexpressing breast cancers in both metastatic and neoadjuvant settings have not been reported in Chile.

The aim of our study was to assess the effect of combined anti-HER2 treatment in neoadjuvant setting and to identify factors that could affect the therapeutic response of patients diagnosed with breast cancer and HER2 overexpression treated at the Instituto Oncológico Fundación Arturo López Pérez (FALP).

\section{Method}

A case-control study was designed to evaluate the effect of clinical and histopathological variables on the response to combined neoadjuvant therapy with Trastuzumab and Pertuzumab associated with cytotoxic chemotherapy in women with breast cancer and HER2 overexpression attended at FALP between the years 2017 and 2020.

All women with diagnosis of breast cancer and HER2 overexpression confirmed by the fluorescence in situ hybridization (FISH) technique, without metastatic disease and who underwent combined anti-HER2 therapy in neoadjuvant setting in our institution between the mentioned years were included in the study. All patients also received cytotoxic chemotherapy.

Cases were those women who achieved $\mathrm{pCR}$ (ypTO/isNO) at surgery after neoadjuvant chemotherapy (NACT) and controls were those who did not.

The exposure variables evaluated were age, body mass index (BMI), duration and type of neoadjuvant therapy, staging, T and N (cTNM) category [10], maximum tumour size at diagnosis and axillary involvement by ultrasonography. Histopathology of the core biopsy considered the molecular profile (pure HER2 versus HER2/Luminal), oestrogen receptor (ER) and progesterone receptor (PR) expression, Ki67 index (dichotomised as > or <20\%), HER2 copy number and HER2/centromere ratio reported by FISH study. Every patient with HER2 2+ or 3+ was confirmed by FISH.

The response variable was whether or not a complete pathologic response was achieved at surgery after combined neoadjuvant therapy. 
Statistical analysis was performed with R software version 4.0.5 (R Core Team, 2018. Vienna, Austria). The effect of each exposure variable on pathologic response was evaluated using the Wilcoxon sign test for continuous variables, Fisher's exact test for nominal variables and the Cochran-Armitage test for ordinal variables. The combined effect of the exposure variables was then evaluated by multiple logistic regression and the StepWise algorithm was applied to determine which variables best predict the complete pathological response for inclusion in the final model. A model with dichotomous molecular profiling (pure HER2 versus HER2/Luminal) and a second model with molecular profiling broken down into pure HER2, HER2/Luminal ER (+) and HER2/Luminal ER (+) and PR (+) were generated. The magnitude of the effect of the exposure variables on the response variable is presented with Odds Ratio (OR).

Finally, considering that only in 39 patients we were able to obtain the HER2 copy number and HER2/centromere ratio described in the FISH study, the association between this variable and pathologic response was analysed univariate.

\section{Results}

Ninety-four women with non-metastatic breast cancer and HER2 overexpression received neoadjuvant combination chemotherapy with Trastuzumab and Pertuzumab at FALP during the period 2017-2020. Figure 1 shows that $70 \%$ of all patients achieved complete pathologic response. Table 1 shows the demographic, clinical, and anatomopathological characteristics of the group analysed.

Table 2 compares demographic, clinical, and histopathologic variables of patients with complete pathologic response (cases) and those without complete pathologic response (controls). We highlight a higher proportion of women with cancer in more advanced stages and with Ki67 $>20 \%$ when $p C R$ was achieved.

When developing the first multiple logistic regression model and applying the StepWise algorithm (Table 3), it was found that the OR of not having a complete pathological response in women with HER2/Luminal breast cancer was 8.15 with respect to the pure HER2 profile (95\% $\mathrm{Cl}$ : 2.43-37.7), while the OR for clinical stage change (I, II, III) was 0.28 (95\% Cl: 0.06-1.05).

When developing the multiple logistic regression model with the molecular profile broken down into pure HER2 (base level), HER2/Luminal with ER (+) and HER2/Luminal ER (+) and PR (+) and applying the StepWise algorithm (Table 3), it was found that the latter profile with expression of both receptors had an OR of not having a complete pathological response of 10.42 (95\% Cl: 3.03-47.9).

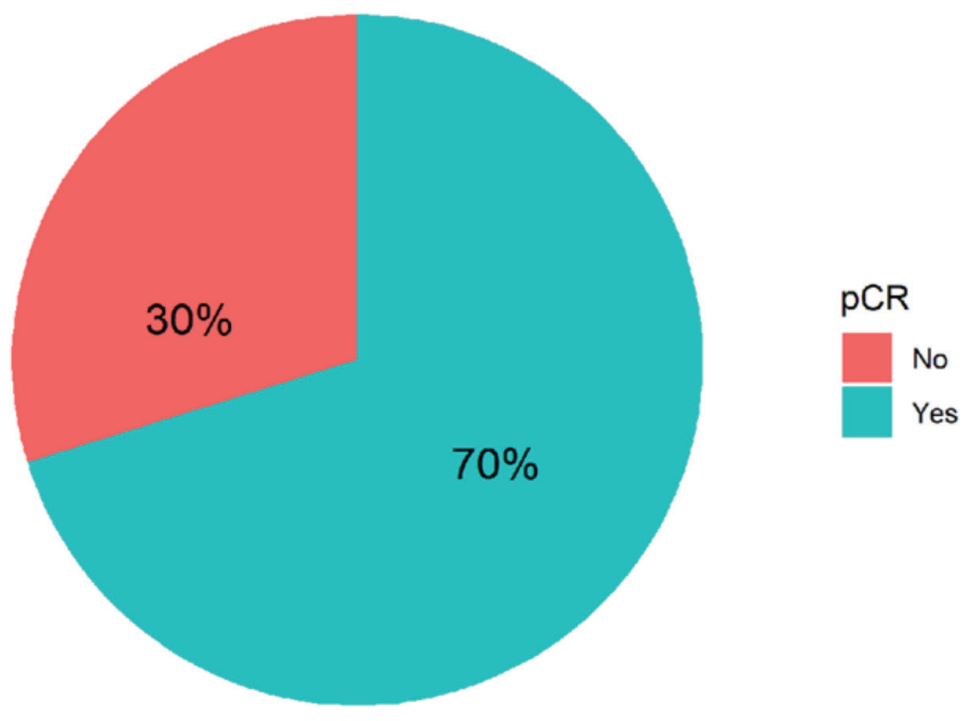

Figure 1. Types of pathological response in 94 women with breast cancer and HER-2 overexpression treated with NACT plus double anti-HER2 blockade at FALP in the period 2017-2020. 
Table 1. Demographic, clinical and histopathological variables in 94 women with breast cancer and HER2 overexpression treated with neoadjuvant at FALP between 2017 and 2020.

\begin{tabular}{|c|c|}
\hline Variables & $N=94$ \\
\hline Median age (range) & $53(32-77)$ \\
\hline \multicolumn{2}{|l|}{ HER2 (\%) } \\
\hline Pure & $36(38 \%)$ \\
\hline Luminal & $58(62 \%)$ \\
\hline \multicolumn{2}{|l|}{ cT (\%) } \\
\hline $\mathrm{T} 1$ & $13(14 \%)$ \\
\hline $\mathrm{T} 2$ & $55(59 \%)$ \\
\hline T3 & $19(20 \%)$ \\
\hline T4 & $6(6 \%)$ \\
\hline Not registered & $1(1 \%)$ \\
\hline \multicolumn{2}{|l|}{$\mathrm{cN}(\%)$} \\
\hline NO & $46(49 \%)$ \\
\hline N1 & $28(30 \%)$ \\
\hline N2 & $12(13 \%)$ \\
\hline N3 & $4(4 \%)$ \\
\hline Not registered & $4(4 \%)$ \\
\hline \multicolumn{2}{|l|}{ Chemotherapy scheme (\%) } \\
\hline TCH-P & $91(95 \%)$ \\
\hline Others & $5(5 \%)$ \\
\hline \multicolumn{2}{|l|}{ Breast surgery (\%) } \\
\hline Partial or consertvative mastectomy & $50(53 \%)$ \\
\hline Total or radical mastectomy & $44(47 \%)$ \\
\hline \multicolumn{2}{|l|}{ Types of pathologic response (\%) } \\
\hline Incomplete & $28(30 \%)$ \\
\hline Complete & $66(70 \%)$ \\
\hline
\end{tabular}

Finally, when comparing the mean value of HER2/centromere ratio (Figure 2a) and HER2 copy number (Figure 2b) described in FISH with the $\mathrm{pCR}$ rate, both values were significantly higher in patients with $\mathrm{pCR}(\mathrm{W}=59, p$-value $=0.003032)$.

In 50/94 cases (53\%), breast-conserving surgery (partial mastectomy) was performed after combined neoadjuvant therapy. Figure 3a shows the relative importance of local extension strata in the breast (cT) before neoadjuvant therapy in partial mastectomies. In 58/94 (62\%) cases, conservative axillary surgery (exclusively sentinel node biopsy) was performed after combined neoadjuvant therapy. Figure 3b shows the relative importance of nodal involvement strata $(\mathrm{cN})$ before neoadjuvant therapy in sentinel node biopsies without axillary dissection.

\section{Discussion}

The 70\% of pCR reported in this study with six cycles of TCHP (Carbotaxol + Trastuzumab + Pertuzumab) is somewhat higher than that reported in the TRYPHAENA clinical trial in which case this percentage was 64\%. This is the first data obtained in Chile as a result of double 
anti-HER2 blockade in neoadjuvant treatment with real-world patients. In the case of patients with negative immunohistochemistry for hormone receptors, the rate of pCR was significantly higher (89\%), also in agreement with international data and somewhat higher than in the TRYPHAENA study, whose rate of pCR in this group was $84 \%$. It can be inferred that the tumour heterogeneity of HER2/Luminal breast cancer conditions a lower response to neoadjuvant therapies, so much so that the multivariate analysis indicates that the risk of not achieving pCR is 8 times higher in these patients than in those with pure HER2 or hormone receptor-negative tumours. Moreover, having both hormone receptors positive (oestrogen and progesterone), which would speak of an even more luminal tumour, raises the OR of not having pCR to 10 times. This also supports the results found in previous phase 2 studies.

In surgery, the trend over time has been to perform less invasive procedures that have less long-term morbidity, without compromising oncologic safety. The benefit of neoadjuvant treatments in achieving breast conservation is well known, and de-escalation in axillary surgery in patients with positive and negative axilla has been validated [11].

With respect to breast surgery, although it is true that there are conditions that contraindicate breast conservation such as multicentric tumours or tumours with extensive inflammatory and/or local involvement, the tendency is to attempt partial mastectomy. Chemotherapy is a tool that facilitates breast-conserving surgery when there is tumour response, especially in T3 or T2 tumours with poor breast-tumour ratio and that do not allow an adequate cosmetic result. Conservative surgery was performed post NACT in $53 \%$ of the cases. Of these, $12 \%$ were T3-T4 and 69\% were T2. It is necessary to mention that other factors to take into account when deciding on conservative surgery are the patient's desire, the feasibility of performing subsequent radiotherapy and the presence of mutations in high-risk genes for breast cancer.

Table 2. Demographic, clinical and histopathological variables in 94 women with breast cancer and HER2 overexpression treated with combined neoadjuvant at FALP between 2017 and 2020 according to pathological response post-surgery.

\begin{tabular}{|c|c|c|c|}
\hline & $\begin{array}{l}\text { No } p C R \\
N=28\end{array}$ & $\begin{array}{c}\text { pCR } \\
N=66\end{array}$ & $p$-value \\
\hline Age $\left(\right.$ years) ${ }^{\mathrm{a}}$ & $50(32-72)$ & $55(32-77)$ & 0.3595 \\
\hline $\mathrm{BMl}^{\mathrm{a}}$ & $28.3(17.8-51.6)$ & $27.9(20.4-38.3)$ & 0.9019 \\
\hline $\begin{array}{l}\text { Treatment duration } \\
\text { (days) }^{a}\end{array}$ & $105.5(71-167)$ & $105(50-306)$ & 0.9206 \\
\hline $\begin{array}{l}\text { TCH-P scheme } \\
\text { chemotherapy (\%) }\end{array}$ & $96.43 \%(27)$ & $96.97 \%(64)$ & 1 \\
\hline Tumour sizea(mm) & $24(6-90)$ & $26(3-80)$ & 0.544 \\
\hline Axillary involvement (\%) & $44.44 \%(12)$ & $53.85 \%(35)$ & 0.4943 \\
\hline \multicolumn{4}{|l|}{ Stage (\%) } \\
\hline I & $44.44 \%(4)$ & $55.56 \%(5)$ & \\
\hline II & $34.48 \%(20)$ & $65.52 \%(38)$ & \\
\hline III & $14.81 \%(4)$ & $85.19 \%(23)$ & $<0.05$ \\
\hline \multicolumn{4}{|c|}{ Molecular subtype and Ki index (\%) } \\
\hline $\begin{array}{l}\text { HER2 } \\
\text { pure }\end{array}$ & $11.11 \%(4)$ & $88.89 \%(32)$ & \\
\hline $\begin{array}{r}\text { HER } 2 \\
\text { Luminal }\end{array}$ & $41.38 \%(24)$ & $58.62 \%(34)$ & $<0.05$ \\
\hline Ki67 $<20$ & $66.67 \%(4)$ & $33.33 \%(2)$ & \\
\hline Ki67 > 20 & $27.27 \%(24)$ & $72.73 \%(64)$ & 0.0624 \\
\hline
\end{tabular}

${ }^{\mathrm{a}}$ Continuous variables are presented as median and range 
Table 3. Effect of clinical and molecular variables on the risk of failing to achieve pCR in a logistic regression model in which hormone receptor expression was dichotomised (HER 2 Luminal versus HER2 Pure) and a logistic regression model in which $(\operatorname{ER}(+) / P R(+), \operatorname{ER}(+), \operatorname{PR}(+)$ and ER (-)/PR (-)) was disaggregated (ER (+)/PR (+), ER (+), PR (+) and ER (-)/PR (-)).

\begin{tabular}{|l|c|c|}
\hline Variables & $\begin{array}{c}\text { OR (risk of not } \\
\text { achieving pCR) }\end{array}$ & $95 \% \mathrm{Cl}$ \\
\hline Stage III versus II & 0.28 & $0.067-1,051$ \\
\hline Ki67 $>$ 20 versus Ki67 <20 & 0.19 & $0.018-1.283$ \\
\hline HER2 luminal versus HER2 pure & 8.15 & $2.429-37.728$ \\
\hline ER (+)/PR (+) versus ER (-)/PR (-) & 10.42 & $3.026-47.858$ \\
\hline
\end{tabular}

(a)

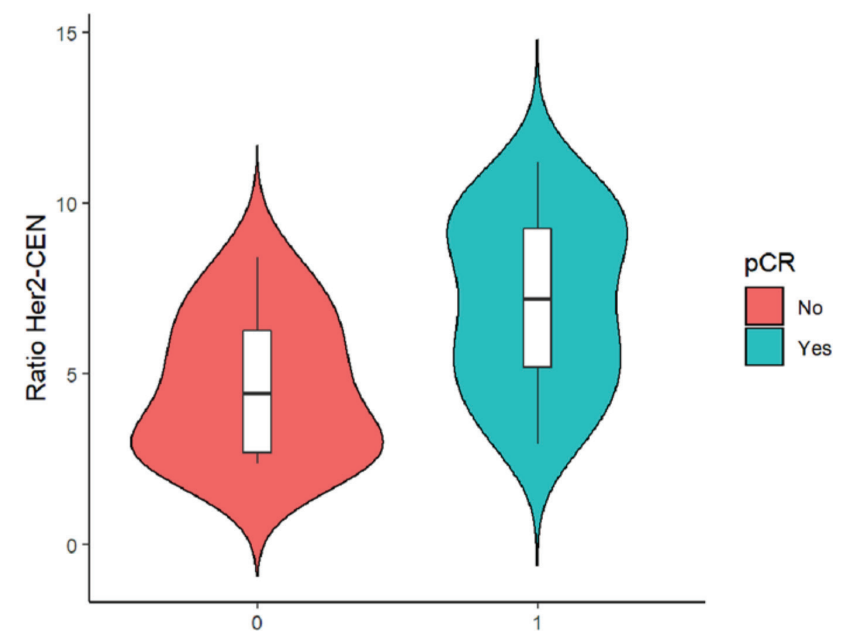

(b)

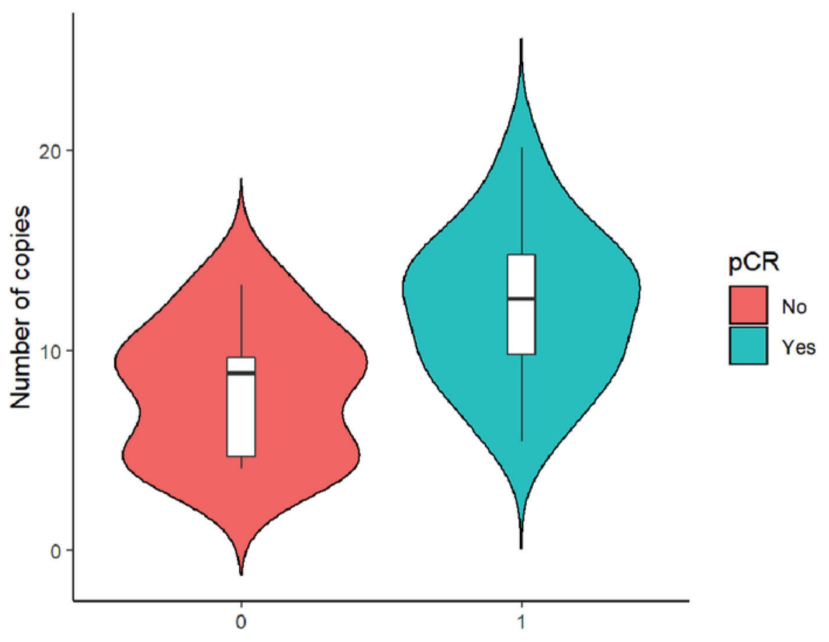

Figure 2. (a): HER 2/centromere ratio value on FISH of core biopsy of women with breast cancer and HER 2 overexpression, who received combined neoadjuvant therapy at FALP in the period 2017-2020, according to pathological response at surgery. Both groups were compared by Wilcoxon sign test, resulting in a statistic $W=-2.84$ and a $p$-value $=0.0037$. (b): HER2 copy number on core biopsy FISH of women with breast cancer and HER 2 overexpression, who received combined neoadjuvant therapy at FALP in the period 2017-2020, according to pathologic response at surgery. Both groups were compared by Wilcoxon sign test, resulting in a statistic $W=78, p$-value $=0.004723$. 
Of the 58 cases in which sentinel lymph node biopsy was performed post NACT, 22 were previous N1, so in 38\% of patient de-escalation was achieved in axillary surgery. It is important to mention that in our institution, we use a double technique to identify the sentinel lymph node post-NACT and also remove the lymph node or nodes marked with a metal clip, which were positive prior to treatment. This has achieved a false negative rate of less than $10 \%$ in N1-2 patients prior to therapy according to international studies, extracting at least three lymph nodes $[12,13]$.

The results obtained in relation to the association of pCR, HER2/CEN17 ratio elevation and HER2 copy number increase add to the emerging evidence, which differs from the results observed in the first large-scale studies of anti-HER2 therapy. Examples of this are the HERA [14] and NSABP B-31 [15] studies, which found no significant association between these parameters and disease-free survival. More recent studies have shown a possible predictive role for pCR. Arnould et al [16] showed in a multivariate analysis that after neoadjuvant therapy patients with high HER 2 amplification ( $>10$ copies/core) had higher pCR than those with low amplification ( $<6$ copies/core) with neoadjuvant therapy with Trastuzumab and taxanes. Similar results were obtained by Choi et al [17], with pCR in tumours with a mean HER2/centromere ratio of 7.08 and an average $>17$ copies HER2/cell.


Figure 3. Types of surgery performed after NACT on (a): breast and (b): axilla in patients with breast cancer and HER2 overexpression at FALP between 2017 and 2020. 
In developing this idea, it is possible to hypothesise that a possible cause for not finding a significant relation previously, may be due to the evolution and sophistication of FISH image capture systems, as well as the improvement of processing protocols, currently allowing to obtain high quality images and easy interpretation, unification and greater clarity of the criteria for assessing the results of FISH.

The limitations of our studies include a small sample size, which did not allow us to include more variables in the regression models, and not having the data to calculate the HER2/centromere ratio and the number of HER2 copies in all cases.

\section{Conclusions}

This study provides real-world data on the effect of dual HER-2 blockade in our population of breast cancer patients with HER-2 overexpression. The $\mathrm{pCR}$ rates with this treatment strategy are high and comparable to what has been described internationally. To explore the association of $\mathrm{pCR}$ with other variables and whether the absence of residual invasive disease post-HER-2 treatment correlates in our centre with an improvement in progression-free survival and overall survival is a matter to be investigated in the future. In our patients as described in other studies, the absence of hormonal receptor expression is a strong predictor of complete pathologic response. We still need more data to show the correlation between pCR, HER2 copy number and HER2/centromere ratio.

The analysis of our results could contribute to the design of public health policies that incorporate high-cost treatments.

\section{Funding}

Financial support for data collection and publication fees was obtained from Roche Laboratory.

\section{Conflicts of interest}

Consulting fees and contracted research for Novartis and Roche.

\section{References}

1. Sung H, Ferlay J, and Siegel RL, et al (2021) Global cancer statistics 2020: GLOBOCAN estimates of incidence and mortality worldwide for 36 cancers in 185 countries CA Cancer J Clin 71 209-249 https://doi.org/10.3322/caac.21660

2. The Global Cancer Observatory-All Rights Reserved, March 2021

3. Waks A, and Winer E (2019) Breast cancer treatment review JAMA 321(3) 288-300 https://doi.org/10.1001/jama.2018.19323

4. Piccart-Gebhart MJ, Procter M, and Leyland-Jones B, et al (2005) Herceptin adjuvant (HERA) trial study team Trastuzumab after adjuvant chemotherapy in HER2-positive breast cancer N Engl J Med 353(16) 1659-1672 https://doi.org/10.1056/NEJMoa052306

5. Baselga J, Gelmon KA, and Verma S, et al (2010) Phase II trial of pertuzumab and trastuzumab in patients with human epidermal growth factor receptor 2-positive metastatic breast cancer that progressed during prior trastuzumab therapy J Clin Oncol 28 $1138-1144$ https://doi.org/10.1200/JCO.2009.24.2024

6. Gianni L, Pienkowski T, and Im YH, et al Efficacy and safety of neoadjuvant pertuzumab and trastuzumab in women with locally advanced, inflammatory, or early HER2-positive breast cancer (NeoSphere): a randomized multicentre, open-label, phase 2 trial Lancet Oncol 13 25-32 
7. Schneeweiss A, Chia S, and Hickish T, et al (2013) Pertuzumab plus trastuzumab in combination with standard neoadjuvant anthracycline-containing and anthracycline-free chemotherapy regimens in patients with HER2-positive early breast cancer: a randomized phase II cardiac safety study (TRYPHAENA) Ann Oncol 24 2278-2284 https://doi.org/10.1093/annonc/mdt182 PMID: 23704196

8. Baselga J, Cortés J, and Kim SB, et al (2012) Pertuzumab plus trastuzumab plus docetaxel for metastatic breast cancer N Engl J Med 366 109-119 https://doi.org/10.1056/NEJMoa1113216

9. Swain SM, Baselga J, and Kim SB, et al (2015) Pertuzumab, trastuzumab, and docetaxel in HER2-positive metastatic breast cancer N Engl J Med 372 724-734 https://doi.org/10.1056/NEJMoa1413513

10. Edge SB, Byrd DR, and Compton CC, et al (2010) AJCC Cancer Staging Manual 7th edn (France: Springer) [http://www.springer.com/ medicine/surgery/book/978-0-387-88440-0] Date accessed: 12/06/14

11. Pilewskie M, and Morrow M (2017) Axillary nodal management following neoadjuvant chemotherapy: a review JAMA Oncol 3(4) 549555 https://doi.org/10.1001/jamaoncol.2016.4163 PMCID: 5580251

12. Boughey JC, Suman VJ, and Mittendorf EA et al (2013) Alliance for clinical trials in oncology Sentinel lymph node surgery after neoadjuvant chemotherapy in patients with node-positive breast cancer: the ACOSOG Z1071 (Alliance) clinical trial JAMA 310(14) 1455-1461 https://doi.org/10.1001/jama.2013.278932 PMID: 24101169 PMCID: 4075763

13. Boileau JF, Poirier B, and Basik M et al (2015) Sentinel node biopsy after neoadjuvant chemotherapy in biopsy-proven node-positive breast cancer: the SN FNAC study J Clin Oncol 33(3) 258-264 https://doi.org/10.1200/JCO.2014.55.7827

14. Piccart-Gebhart MJ, Procter M, and Leyland-Jones B et al (2005) Trastuzumab after adjuvant chemotherapy in HER2-positive breast cancer N Engl J Med 353(16) 1659-1672 https://doi.org/10.1056/NEJMoa052306 PMID: 16236737

15. Tan-Chiu E, Yothers G, and Romond E et al (2005) Assessment of cardiac dysfunction in a randomized trial comparing doxorubicin and cyclophosphamide followed by paclitaxel, with or without trastuzumab as adjuvant therapy in node-positive, human epidermal growth factor receptor 2-overexpressing breast cancer: NSABP B-31 J Clin Oncol 23(31) 7811-7819 https://doi.org/10.1200/ JCO.2005.02.4091

16. Arnould L, Arveux P, and Couturier J et al (2007) Pathologic complete response to trastuzumab-based neoadjuvant therapy is related to the level of HER-2 amplification Clin Cancer Res 13(21) 6404-6409 https://doi.org/10.1158/1078-0432.CCR-06-3022 PMID: 17975153

17. Choi JH, Jeon CW, and Kim YO, et al (2020) Pathological complete response to neoadjuvant trastuzumab and pertuzumab therapy is related to human epidermal growth factor receptor 2 (HER2) amplification level in HER2-amplified breast cancer Medicine (Baltimore) 99(46) e23053 https://doi.org/10.1097/MD.0000000000023053 